\title{
The Lox Gene Expression in the Breast Cancer Tumor Tissue and Its Relationship with Cilinopathological Markers
}

\author{
Narjes Tavakoli Pashkami ${ }^{1,}{ }^{,}$, Fourozandeh Mahjoobi ${ }^{1}$, Tayebe \\ Majidzadeh ${ }^{1}$, Banafsheh Afkari Eidehlo ${ }^{2}$ \\ ${ }^{1}$ Department of Medical Genetics, National Institute for Genetic Engineering and \\ Biotechnology, Tehran, Iran \\ ${ }^{2}$ Department of Biology, Faculty of Science, Nour-e-Danesh Institute of Higher \\ Education, Meymeh, Isfahan, Iran \\ * Corresponding author: Narjes Tavakoli Pashkami, Department of Medical Genetics, \\ National Institute for Genetic Engineering and Biotechnology, Tehran, Iran. E-mail: \\ n_tavakoli89@yahoo.com
}

DOI: $10.21859 /$ mci-supp-28

\section{Keywords:}

Breast cancer

Lox gene

Biomarker

Gene expression

\begin{abstract}
Introduction Breast cancer is a common cancer of women. Although significant scientific advancements have been made in the field of prevention and treatment, this information still does not offer the influential ability in reducing mortality. The study of the expression of genes that have been altered in cancer can be used as an effective biomarker for better managing for disease. The lox gene encodes a lysine oxidase which naturally increases the elasticity of the tissue. Recent studies showed lox's intervention in more important biological activities. The lox gene induces movement and migration in cells. It plays a role in cell signaling and transcription of genes. This means that lox gene incorrect expression may lead to tumor progression and metastasis. Then this gene can be considered as a probable biomarker in the prognosis of the pathway of the disease.

Materials and Methods: 70 samples, including 35 tumor samples and 35 healthy adjacent samples to the tumor, were collected from patients with breast cancer after surgery. After extracting RNA and synthesizing cDNA, using the Real-Time PCR method, the lox gene expression and the B-actin gene as huosekeeping gene were measured. Then, changes in the lox gene expression in tumor tissues were investigated in comparison with the normal tissues.

Results: The RT-PCR produced a 106bp product, as expected to fit the lox design piece. Real-time PCR results showed an increase in the lox gene expression in the tumor tissues compared with the normal tissues adjacent to the tumor.

Conclusions: Statistical analysis revealed that the lox gene expression had a significant increase in the tumor tissues compared to the healthy tissues. Taking the findings into consideration, it is highly likely that this gene can be considered as a candidate for proper biomarkers for prognosis and prediction of progression of breast cancer.
\end{abstract}

